\title{
Statistical survey and comprehensive review on human skin detection
}

\author{
Hussein Ali Hussein Al Naffakh ${ }^{1}$, Rozaida Ghazali², Nidhal Khdhair El Abbadi ${ }^{3}$ \\ ${ }^{1,2}$ Faculty of Computer Science and Information Technology, Universiti Tun Hussein Onn Malaysia, Malaysia \\ ${ }^{3}$ Faculty of Education, University of Kufa, Iraq
}

\begin{abstract}
Article Info
Article history:

Received Mar 8, 2020

Revised May 24, 2020

Accepted Jun 30, 2020

Keywords:

Color space

Dataset

Skin color modeling

Skin detection

Skin segmentation

ABSTRACT

With the advancement of data society today, pictures have turned out to be increasingly imperative. Automatic detection of human skin has been an area of active research for the past few years. Human skin detection assumes a vital job in a wide scope of picture preparing applications going from face detection, steganography, face tracking, age detection, discover pornographic images, the discovery of skin diseases gesture analysis and substance based picture recovery frameworks and to different human PC association spaces. Detecting human skin in complex pictures have ended up being a difficult issue since skin shading can fluctuate drastically in its appearance because of numerous variables, for example, illumination, race, maturing, imaging conditions, and complex foundation. In this study, we will study and analyze skin researches, where we will treat the weakness of previous research of survey on human skin detection methods. The reason for this investigation is to give a state-of-the-art study on human skin molding and detection methods in 1998-2019 periods. Furthermore, this research presented the statistical study for each issue stated before. We finish up with a few ramifications for a future course. Study results will benefit all researchers who are interested in human skin detection topic.
\end{abstract}

This is an open access article under the CC BY-SA license.

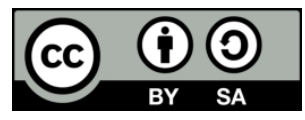

\section{Corresponding Author:}

Hussein Ali Hussein Al Naffakh,

Faculty of Computer Science and Information Technology,

Universiti Tun Hussein Onn Malaysia,

86400 Parit Raja, Johor, Malaysia.

Email: aulanone@gmail.com

\section{INTRODUCTION}

Human skin detection is among the most commonly applied algorithms in visual field literature and has been used directly and indirectly in numerous applications. Some attention has been paid to the field of face analysis and human detection, tracking and recognition systems. It has proven intimidating to detect human skin in complex images because the appearance of human skin may vary significantly due to a number of factors including illumination, ethnicity, hair effect, imagery and complexity background. Researchers have struggled over these difficulties over the past twenty years, leading to hundreds of articles being published. The fast-growing research to detect human skin is built around the idea of extracting information from colorful images about individuals, intentions, mode and image and computer content and then being able to react appropriately.

There are some survey papers reviewed on human skin detection [1], skin detection in colored images [2] and skin tone detection using color spaces [3]. This paper is aimed at collecting information about human skin detection techniques in literature and to summarize key ideas and issues in human skin detection. We attempted to summarize the most important and remarkable distinctions between these methods, their strengths and limitations, and characteristics. This research also studied color spaces, challenges, real-time 
system, datasets and measurements in statistical, threshold, region and machine learning methods researches. This research presented the statistics of study as well.

Skin color appearance provides us a sign of the person's ethnicity, health, and age. Human skin contains of three primary epidermis, dermis and subcutaneous layers [4]. Every layer has a purpose and works together on every layer. The skin surface is coated by dead skin cells that do not reflect regularly while sweat or skin oil causes skin gloss. The encounters between light and skin can lead to complex phenomena $[5,6]$. A combination of skin and light is initial spectrum of the skin: the layers transmit, absorb and reflect the skin. Human skin spectrums normally form an ongoing series of homologues due to the characterization of absorbed melanin and hemoglobin, where melanin plays the dominant role in dermis in the spine and hemoglobin and mainly determines the skin appearance [4, 7]. The appearance of human skin color may differ dramatically with a variety of image types and sources, making accurate skin detection a challenging task. The skin detection challenges can be assigned to certain variables such as differences in illumination, ethnicity, image conditions, image montage and reproduction, cosmetics, maturing, and compound landscape.

In real-world situations, the most important issue is the variation in illumination that severely degrades segmentation efficiency [5]. Changing the spread of light sources or light levels (indoor, outdoor, highlights, shades, non-white lights) results in a skin color shift. The dark shadow on the face is usually the lead of powerful directional illumination that partially obscured certain facial regions. This is because of the non-plane form of the facial characteristics. Occasionally, a face has a "bright spot" due to heavy lighting reflection. In some other drawback, the appearance of skin color differs from person to person caused by physical variation between groups of human races. For instance, Europeans (Caucasians), Africans, Asians, etc., have distinct skin colors ranging from white to brown to dark [8].

Once the picture is developed, variables including lens features (sensor reaction, lenses) influence the skin appearance. Overall, separate color cameras do not automatically create the same color for the same scene in the same picture circumstances [9, 10]. In addition, various internet image collections, films, newspapers, and scanned pictures are typically unmanageable and have almost infinite assembly processes. Using color transfer technique, tools are available to replicate skin colors, including setting fresh pigment concentration and altering skin color image. Some images have already been taken using color filters. This makes it even harder to handle color information. Besides that, makeup changes color of the skin [11]. Human skin differs from smooth, flexible skin to dry, tough skin with wrinkles. For example, furniture, clothing, blonde hair, rocks, etc. For example, furniture, clothing, blonde hair, rocks, and so on. The variety of backgrounds is substantially limitless. This leads to incorrect detections by the skin sensor [11].

In subsequent sections, section 2 highlights some of the related work of skin detection application in multiple disciplines. Section 3 is dedicated to explain all datasets that have been mentioned in this survey. Section 4 briefly explains the type and number of journals for each paper that have been mentioned in this paper. Section 5 describes skin detection methods. In section 6 discussion and future work.

\section{RELATED WORKS}

Researchers have been exploring human skin detection, detecting skin and non-skin pixels. In addition, there are a few studies in this field that compile current developments. In others word, some researchers are focusing on investigating color space with human skin detection methods. An evaluation on human skin detection accuracy using accuracy metrics was presented by Vezhnevets et al. [12]. They attempt to fill this vacuum by examining the methods and techniques most widely used in statistical-based and thresholding-based methods. They evaluated the metrics components as well. However, they did not specify challenges in human skin detection.

A survey of skin-color modeling and detection methods was conducted by Kakumanu et al. [11]. They explore the useful human skin detection techniques for human skin detection tasks of skin pixel and non skin pixel. In their survey they provide the discussion human skin detection techniques. They provided a crucial current review of statistical-based, machine learning-based, thresholding-based and region-based methods based on color information of the visual spectrum. They also mentioned illumination effect but meanwhile not refer to the hair effect.

Another survey on the detection and numerical analysis of skin tones using color space showed an investigation of the most commonly used methods and techniques [3]. This paper focused on pixel-based approaches besides machine learning-based methods. Among various frameworks, statistical approach has been studied and most widely used in skin detection based on color information. Despite that, this paper evaluates skin type's challenges only. To review the work on neural network perspective, Hani K Al-Mohair et al. [13] submitted an extensive study of current work neural network perspective based human skin color detection. The aim of this article is to show different algorithms that used artificial neural network systems to detect skin color, define their techniques and assess their features and achievement. They pointed out the challenges like illumination and hair effect except skin types. 
Mahmoodi [1]'s survey on human skin detection aimed at surveying applications, color spaces, methods and their performance, compensation techniques, and benchmarking datasets on human skin detection, screening relevant research over the past two decades. This paper discussed various challenges and difficulties involved in locating skin pixels. Skin segmentation algorithms are according primarily on color information; a thorough debate on the applicability of divergent color areas is elucidated. In addition, the use of standard evaluation metrics and datasets makes it possible and reasonable to compare methods.

Naji et al. [2]'s survey of skin detection in colored images provided an updated survey of skin color modeling and detection methods. Relevant issues were also discussed, including color spaces, costs and risks, databases, testing and benchmarking. Upon examining these techniques and distinguishing their weaknesses and strengths, they finalized with several possible consequences for future outlook. Their objective is to obtain a latest survey of existing skin detection techniques in this research. The most significant and notable dissimilarity in the capabilities, limitations and characteristics of all these methods has been summarized. In spite of that, this paper failed to address real time system and hair effect.

We sum up the above surveys and current surveys in Table 1 on the content of several aspects of human skin detection. The "generally analyzed" are the topics that are generally identified or addressed. By contrast, "deeply analyzed" are the topics discussed in detail in the articles concerned. From this table it can be seen that our survey has studied in detail the different contents of human skin detection compared to other investigations.

Table 1. Comparison of previous surveys on skin detection and current study

\begin{tabular}{|c|c|c|c|c|c|c|c|c|}
\hline \multirow{2}{*}{ No. } & \multirow{2}{*}{ Comparison attributes and methods } & \multicolumn{7}{|c|}{ Survey papers } \\
\hline & & {$[12]$} & [11] & {$[3]$} & [13] & [1] & {$[2]$} & Current survey \\
\hline \multirow[t]{5}{*}{1.} & \multicolumn{8}{|c|}{ Color Space with Pixel-based skin detection methods: } \\
\hline & Statistical-based methods & $*$ & $* *$ & $* *$ & - & $* *$ & $* *$ & $* *$ \\
\hline & Machine learning-based methods & - & $*$ & - & $*$ & $*$ & $*$ & $* *$ \\
\hline & Thresholding-based methods & $*$ & $*$ & $*$ & - & - & $* *$ & $* *$ \\
\hline & Region-based skin detection methods & - & $*$ & $*$ & - & - & $*$ & $*$ \\
\hline \multirow[t]{3}{*}{2.} & \multicolumn{8}{|c|}{ Evaluation components: } \\
\hline & Data sets & - & $*$ & $*$ & - & $* *$ & $* *$ & $* *$ \\
\hline & Metrics & $*$ & $*$ & $*$ & - & $*$ & $*$ & $*$ \\
\hline \multirow[t]{6}{*}{3.} & \multicolumn{8}{|c|}{ Significance of survey: } \\
\hline & Systematic survey & $*$ & $*$ & - & $*$ & $*$ & $*$ & $* *$ \\
\hline & Discussion & $*$ & $*$ & $*$ & $* *$ & $* *$ & & $* *$ \\
\hline & Significance findings & $*$ & $*$ & $*$ & $*$ & $*$ & $*$ & $* *$ \\
\hline & Future directions & - & - & - & - & $*$ & $*$ & $*$ \\
\hline & Real time system & - & - & - & - & $*$ & - & $* *$ \\
\hline \multirow[t]{4}{*}{4.} & & lenges & & & & & & \\
\hline & Illumination effect & - & $* *$ & - & $*$ & $*$ & $* *$ & $* *$ \\
\hline & Hair effect & - & - & - & $*$ & - & - & $*$ \\
\hline & Skin types & - & - & $*$ & - & $*$ & $* *$ & $* *$ \\
\hline
\end{tabular}

\section{DATASET}

Most skin detection methods obligate a lot of skin and non-skin specimens for training and testing. Overall, a standard test dataset should be used to enable researchers to easily match the results. Different type of dataset has each their unique characteristics which depend on the application. The link for some datasets represented in Table 2, while each characteristic of datasets explained in Table 3.

Table 2. Link of datasets

\begin{tabular}{ll}
\hline \multicolumn{1}{c}{ Type of dataset } & \multicolumn{1}{c}{ Link of dataset } \\
\hline AR & http://rvl1.ecn.purdue.edu/ aleix/aleix_face_DB.html or http://RVL.www.ecn.purdue.edu \\
BAO & https:///facedetection.com/wp-content/uploads/BaoDataBase.zip \\
Caltech & http://www.vision.caltech.edu/html-files/archive.html \\
ECU & https://www.uow.edu.au/ phung/download.html \\
HGR & http://sun.aei.polsl.pl/ mkawulok/gestures/ \\
Ibtd & http:///bmedia.ece.ucsb.edu/resources/dataset/ibtd.zip \\
LFW & http://vis-www.cs.umass.edu//fw/ \\
Michigan Face & http://www.engin.umich.edu/faces \\
MUCT & www.milbo.org/muct \\
SFA & http://www.sel.eesc.usp.br/sfa \\
UCD & http://dsp.ucd.ie/-prag \\
Pratheepan & http://cs-chan.com/project1.htm \\
\hline
\end{tabular}


Table 3. Type of datasets for skin detection

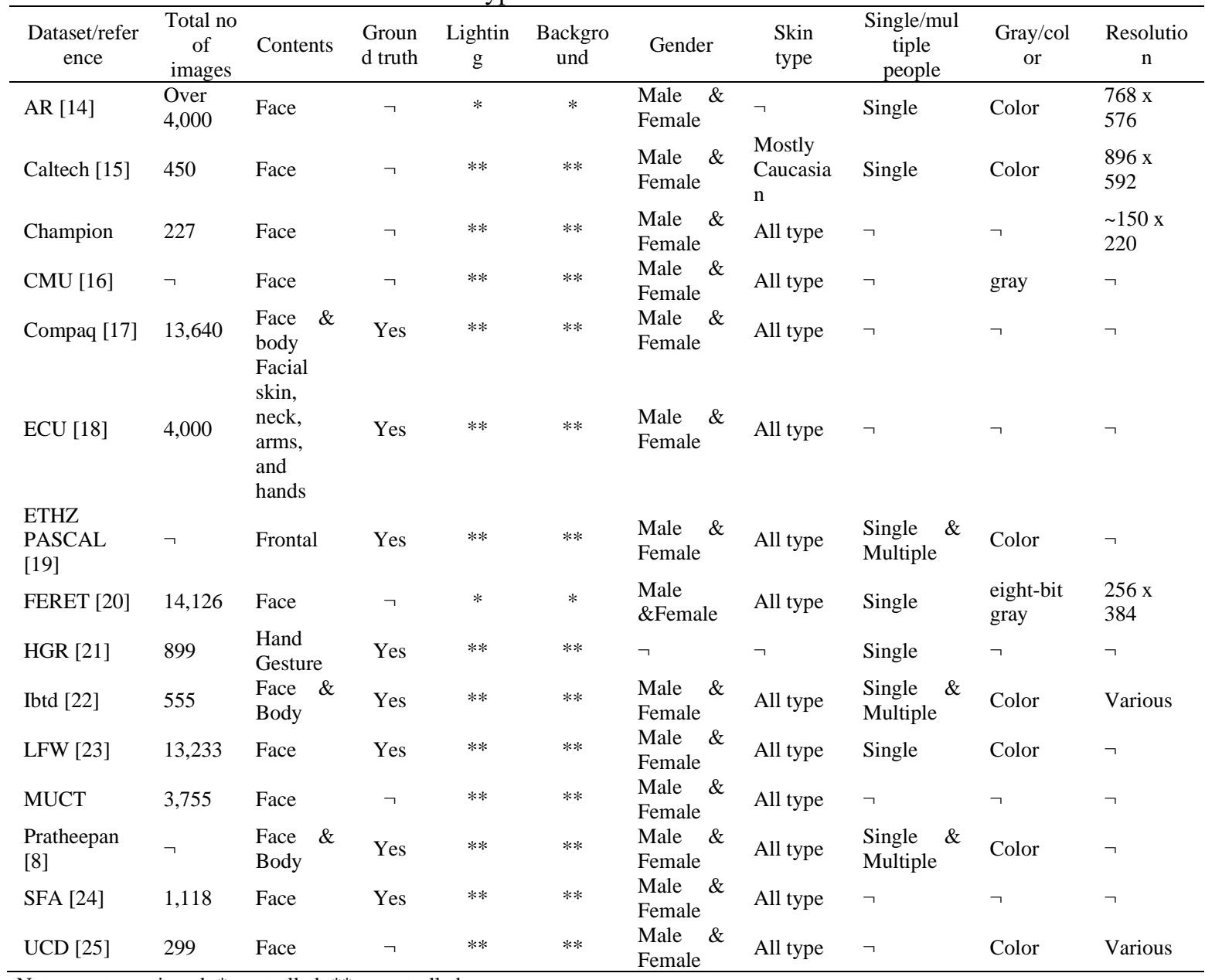

Note: ᄀnot mentioned; * controlled; **uncontrolled

\section{REVIEW PROTOCOL}

For our survey, nine search venues are focused, as shown in Table 4. They are Institute of Electrical and Electronics Engineers (IEEE Xplore), Elsevier, arXiv, World Scientific, The Institution of Engineering and Technology (IET), Springer, Citeseer, Inc, and IGI Global. The related material that was not found at the given nine locations was downloaded with Google Scholar and added to the "Other" category. Human skin detection is one of the most captivating topics in the eyes of researchers, where through Table 4 has been found a large number of researches that has been published in the journals with high reputation. This indicates that this field is useful for researchers whom interests in this field.

Table 4. Type and number of journals

\begin{tabular}{lc}
\hline Name of journal & No. of researches \\
\hline IEEE & 52 \\
Elsevier & 33 \\
Springer & 14 \\
arXiv & 8 \\
World Scientific & 5 \\
Citeseer & 5 \\
IET & 3 \\
Inc & 2 \\
IGI Global & 2 \\
Other & 49 \\
\hline
\end{tabular}




\section{SKIN DETECTION METHODS}

In this segment, we analysis current methods for identifying color image skin regions. We categorize the identification techniques of single images into two classifications; pixel-based methods and region-based methods. Pixel-based skin detection methods categorize each pixel independently as skin or non-skin without taking neighbors into account. The skin detector will inspect color pixels that match the skin color model [26]. These methods are classified into three categories: statistical, machine learningbased and threshold-based methods. Statistical-based methods focus on data collection, study structure, data summary, producing a model to illustrate awareness of how data is linked, and predicting the future (i.e., classification). Statistics can use the companion's probability topic. These methods include: histograms, Lookup tables, distance-based detectors, Bayes theorem, and Gaussian distribution. Machine learning-based methods are attempting to construct skin sensors with the potential to be discovered from data collection training without creating a clear skin color model. Usually these techniques use supervised learning. These methods involve different machine learning techniques, for instance artificial neural networks (ANN), support vector machines (SVM), and fuzzy logic. Thresholding-based methods depict the threshold-oriented skin model using basic classification laws. Typically, multiple threshold levels are used for each color component. Many techniques are suggested to select threshold values. Consumers can select a threshold amount individually and view the outcomes interactively. Trial and error come in and you want the outcome to be as great as it is. Other techniques calculate the value automatically using a thresholding algorithm recognized as automatic thresholding. During the segmentation process, adaptive thresholding method can also be used to dynamically update threshold values.

Region-based skin detection methods spatial data is helpful as most real-life object segments made up of spatially linked pixels. The primary concept of region-based segmentation methods is to distinguish different regions in images with identical characteristics [27]. Approaches based on the region can be classified into: region growing and watershed segmentation. Some studies used a combination of color spaces or hybrid techniques. The upcoming segments discuss the purpose and overall strategy of each method. The statistics of number of researches that used in human skin detection with statistics, threshold, region and machine learning methods for 1998-2019 periods, as can be seen in Figure 1, gives brief information about this topic. There is significant period, 2009-2017, shows that researches in this area are increasing with the highest number of 10 in 2012, whereas, in 2006 and 2018 we could not find any research in this topic.

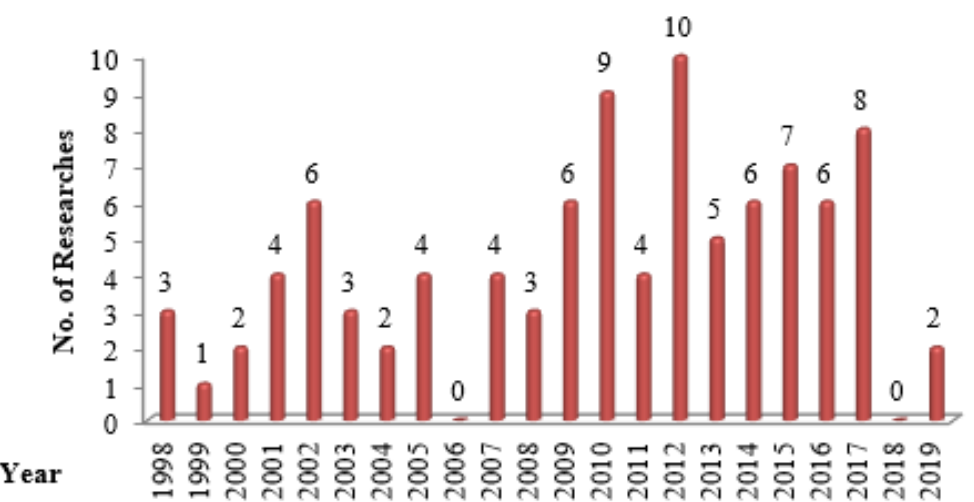

Figure 1. Number of researches from 1998-2019 for skin detection with statistical, threshold, region and machine learning methods

There are more than 20 types of datasets that used in human skin detection from same period. FERET, AR, Pratheepan, and HGR are four datasets which have slightly higher number than most of the dataset as illustrated in Figure 2. As presented, Compaq and ECU are the highest datasets, 18 and 12 respectively, which put to use in this topic. These datasets have full body images with ground truth as mentioned in Table 3. From this, we can draw a conclusion that these datasets are the best used for human skin detection. 


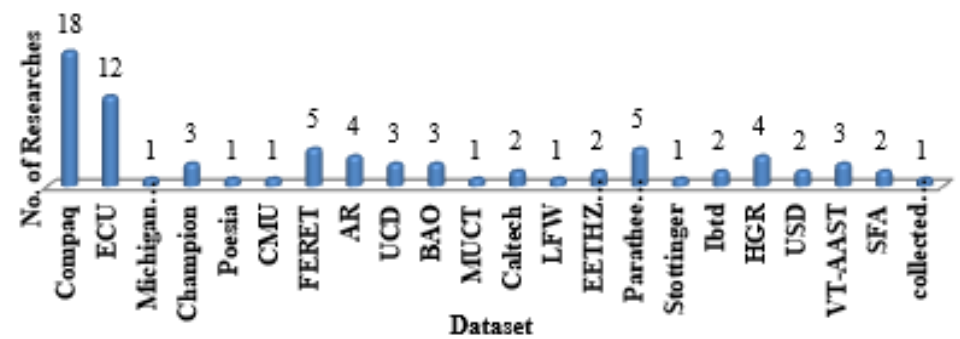

Figure 2. Type of datasets for skin detection with statistical, threshold, region and machine learning methods in 1998-2019

The summary of color spaces that have been used in human skin detection researches within 1998-2019 periods is represented in Figure 3. There are around 10 color spaces used. The most used color spaces are RGB, YCbCr, HSV and CIE with 49, 39, 37, and 18 respectively. RGB is the most basic color space which represented three primary colors. $\mathrm{YCbCr}$ color space is widely used for film and digital photography technologies. HSV space is appropriate for image processing as it separates hue (chrominance) data from luminance (intensity) data. And the last one, CIE offers a color-defining instrument, but it does not directly correspond to hardware operation or human vision.

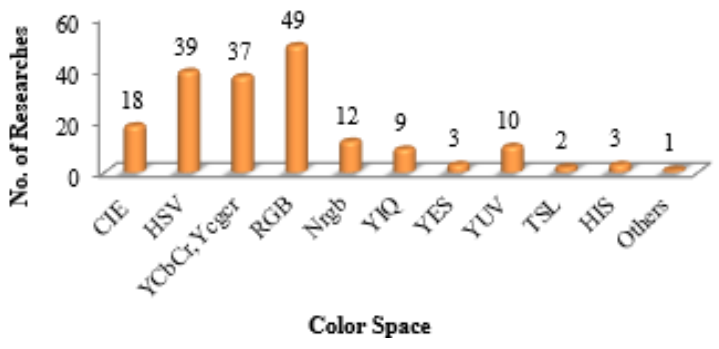

Figure 3. Type of color spaces for skin detection in statistical, threshold, region, and machine learning methods in 1998-2019

Types of statistical, threshold and region methods that have been used in human skin detection researches between 1998-2019 is expressed in Figure 4. Threshold is the most used method with 24 researches. Other than that, GMM, Bayesian and Histogram have more than 10 researches for each. The thresholding technique is the easiest and most popular method of segmentation of images [28]. It can be considered as a quick way to separate items from their background. All of the measurements' types and frequencies of researches that used in measuring the accuracy of human skin detection researches in 1998-2019 periods is represented in Figure 5. With more than 20 measurements Accuracy, FPR, TPR are the most used measurements with 20, 22 and 14mentioned in researches respectively.

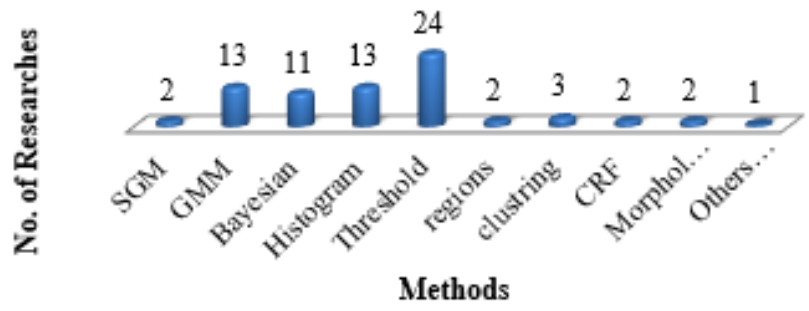

Figure 4. Type of statistical, threshold and region methods in 1998-2019 


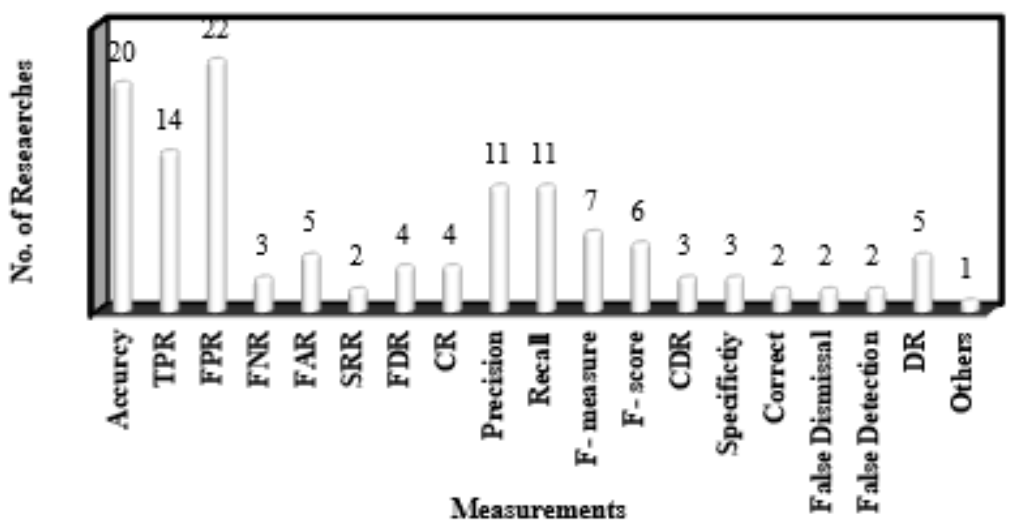

Figure 5. Number of performance matrices used for skin detection researches in statistical, threshold, region, and machine learning methods in 1998-2019

The ratio of researches that mentioned the following challenges; skin types, illumination, hair effect and real time system in statistics, threshold, and region methods in 1998-2019 is represented in Figure 6. As it turns out, from the total of 59 researches, there are $34 \%$ of researches which not mention any of these challenges. Likewise, there are $37 \%, 29 \%$, and $7 \%$ researches for illumination, skin types, and real time system respectively. Whereas, there is no research mention about hair effect challenge. These challenges are one of the major challenges in human skin detection. The results of this study show that a small number of special methods were used to reduce illumination and the rest of the research was used, like space colors, because of their effect on illumination. Thus, this is image is not considered a good treatment for dark, shadow and light illumination and the percentage of error in human skin detection is unsatisfied.

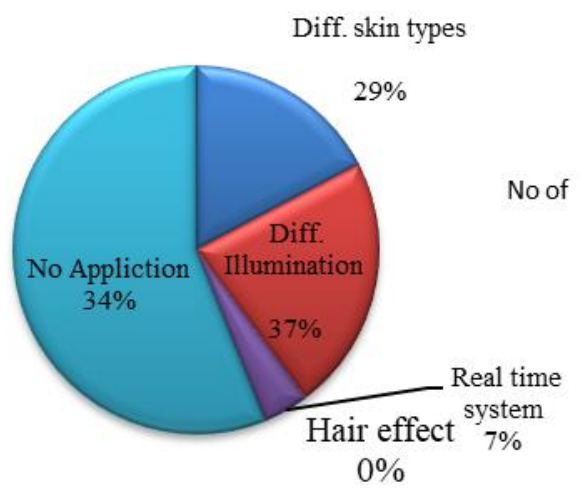

Figure 6. Percentage of researches that considered with different skin types, different illumination, hair effect and real time system in statistical, threshold and region methods in 1998-2019

Ten types of machine learning methods that used in researches in 1998-2019 are described in Figure 7. ANN is the most used method with number of researches is 20. ANN aims to learn unidentified complicated input-output interactions depending on training data and then to be prepared to predict unseen information [13]. Types of training algorithms that used in human skin detection research in same period are portrayed in Figure 8. Back propagation got the highest number of researches by ten among 12 algorithms. 


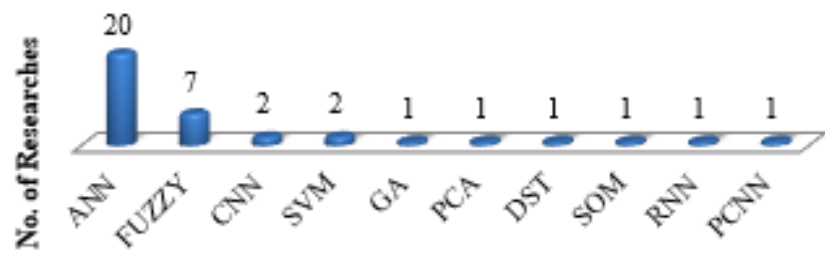

Methods

Figure 7. Type of machine learning methods in 1998-2019

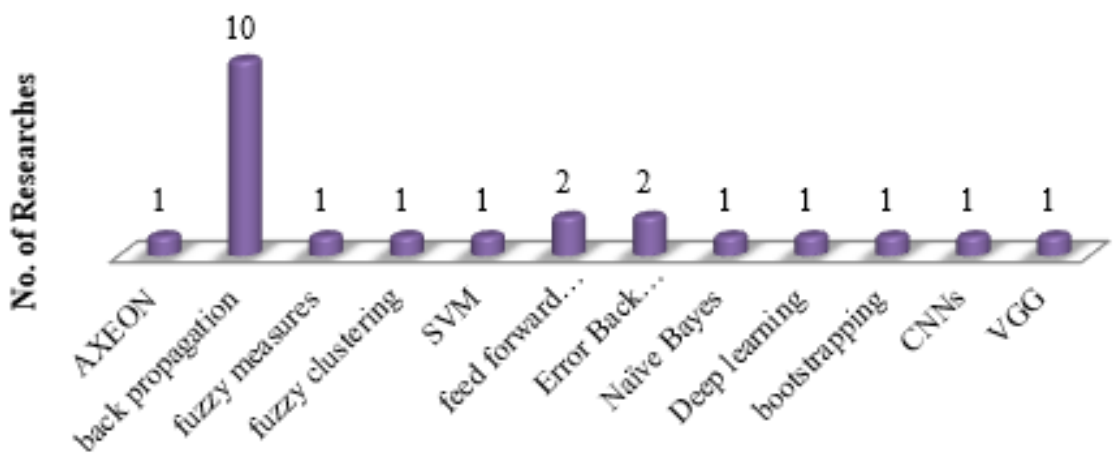

Training algorithm

Figure 8. Type Training algorithms in 1998-2019

The percentage of researches in human skin detection that mentioned challenges; illumination, hair effect, real time system and hair effect, in machine learning methods in 1998-2019 is illustrated in Figure 9. Out of the total of the researches, which is 37, there is $40 \%$ of researches do not mention any of the challenges. While for different skin types, illumination, real time system and hair effect are $14 \%, 27 \%$, $19 \%$, and $0 \%$ respectively.

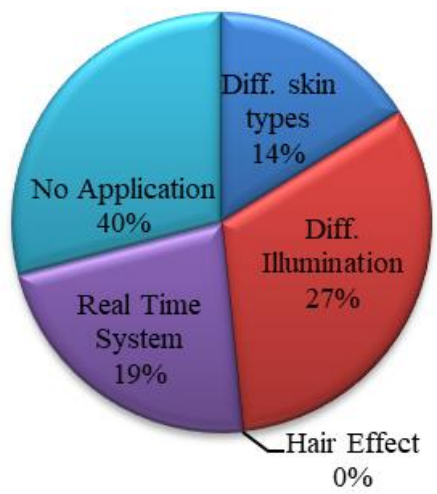

Figure 9. Percentage of researches that considered with different skin types, different illumination, hair effect and real time system in machine learning methods in 1998-2019

The related researches of human skin detection are evaluated in Table 5. Several categories are considered in this evaluation, such as color space, method, measurement, application, and time. A few researchers were evaluated the time consuming of skin detection. However, the others were focused on the confection matrix evaluation. 
Table 5. Real time system of human skin detection methods

\begin{tabular}{|c|c|c|c|c|c|c|}
\hline Reference & $\begin{array}{c}\text { Color } \\
\text { space skin }\end{array}$ & Detection method & $\begin{array}{c}\text { Other detection } \\
\text { involved }\end{array}$ & $\begin{array}{c}\text { Evaluation } \\
\text { metrics }\end{array}$ & $\begin{array}{c}\text { Datasets/application } \\
\text { area }\end{array}$ & Detection time \\
\hline [29] & HSV & $\begin{array}{l}\text { SVM, KNN \& } \\
\text { Bayesian }\end{array}$ & NO & Accuracy & FERET & $(2.66-20.54) \mathrm{S}$ \\
\hline$[30]$ & $\mathrm{YCbCr}$ & $\begin{array}{l}\text { A parametric } \\
\text { ellipse }\end{array}$ & FACE & FP \& DR & Champion & $(9.29-39.93) \mathrm{S}$ \\
\hline [31] & HSV & $\begin{array}{l}\text { Threshold \& } \\
\text { histogram }\end{array}$ & NO & NA & $\begin{array}{l}379 \text { images obtained } \\
\text { from the Internet. }\end{array}$ & $41.68 \mathrm{~s}$ \\
\hline [32] & $\begin{array}{l}\text { RGB, } \\
\mathrm{HSV}, \text { and } \\
\mathrm{YCbCr}\end{array}$ & $\begin{array}{l}\text { Haar-like and } \\
\text { AdaBoost }\end{array}$ & $\begin{array}{l}\text { FACE \& } \\
\text { HAND }\end{array}$ & $\begin{array}{l}\text { FP \& F- } \\
\text { measure }\end{array}$ & FERET & $90 \mathrm{~ms}$ \\
\hline [33] & $\mathrm{YCbCr}$ & $\begin{array}{l}\text { a very fast efficient } \\
\text { pre-processing and } \\
\text { a local two-stage } \\
\text { diffusion method }\end{array}$ & FACE & $\begin{array}{l}\text { Precision, } \\
\text { Recall \& F- } \\
\text { score }\end{array}$ & SDD & $\begin{array}{l}98 \text { frames of } 640 * 480 \\
24 \text {-bit color images per } \\
\text { second. }\end{array}$ \\
\hline [34] & RGB & $\begin{array}{l}\text { Block Approach \& } \\
\text { Threshold }\end{array}$ & FACE & NA & FERET & $(0.203-9.219) \mathrm{S}$ \\
\hline [35] & $\begin{array}{l}\text { RGB } \\
\& Y U V\end{array}$ & FPGA & FACE & NA & NA & $\begin{array}{l}640 \times 480 \text { pixel image } \\
\text { are executed in } 90 \mathrm{~ms}\end{array}$ \\
\hline$[36]$ & HSV & ANN & $\mathrm{NO}$ & Accuracy & $\begin{array}{l}2000 \text { human skin } \\
\text { images were } \\
\text { downloaded from the } \\
\text { internet }\end{array}$ & $(324.22-926.3) \mathrm{S}$ \\
\hline [37] & RGB & Threshold & FACE & FNR \& FPR & NA & $\begin{array}{l}(640 \times 480) \text { with } 75 \\
\text { FPS, }(800 \times 480) \text { with } \\
60 \quad \text { FPS, and } \\
(1024 \times 768) \text { with } 25 \\
\text { FPS. }\end{array}$ \\
\hline
\end{tabular}

\section{RESULTS AND DISCUSSION}

This survey is an effort to present an up-to-date overview of skin detection research methods outlined in over 150 research papers. In an attempt to find any sensitive part of the human body, skin detection seeks to divide the input picture into regions. An enhanced strategy for human skin segmentation with a higher identification rate increases the efficiency of many computer vision systems. This phase is very crucial as image segmentation is perceived to be the first phase in image analysis and therefore the whole achievement or failure of the entire system is largely based on this phase. Although significant development has been produced over the past two decades, there are many opportunities for further work. Researchers need to be alert of the need for a robust skin detector to be effective against diversity in: illumination environments, varying ethnic groups, picture montage and replication, make-up, aging and complicated background.

Where applicable, we observed the overall efficiency of these techniques. That being said, the techniques in question seemed to use various raw data sets (i.e. training samples) that the researchers collected by hand. The volume and performance of the rough data have a significant impact on the skin color clustering model, although different scientists use the same technique. In other phrases, if new raw skin sample data are assembled from another database, the skin color set may shift dramatically. Hitherto, skin detection techniques have been predominantly subjective, allowing scientists to decide on the effectiveness of these techniques due to the shortage of consistency on how to assess the correctness of a specific clustering model and how these techniques are assessed. In particular, most researchers use their own datasets to conduct their tests and simulations. Despite the same raw data, the use of distinct methods for modeling skin color clusters creates distinct classification boundaries (or clustering models).

\section{CONCLUSION}

Skin detection plays an important role in human motion analysis and face detection. It is widely needed in image processing applications ranging from face detection, face tracking, gesture analysis, and content-based image retrieval systems and to various human computer interaction domains. In this article, the state-of-the-art studies on human skin studies for human skin detection based on image processing are reviewed. This research presented the statistical study for each issue stated before. This study includes several sections of investigation. The literature is compared according to the skin detection methods. Some techniques are intended for a particular purpose in their strategy and are therefore unsuitable to adapt to other applications without taking into account some fundamental assumptions. Others have one or more controllable parameters that the user must modify instead of instantly learning from the picture itself. Then, the utilised datasets in the literature are presented. In addition, the used color space in the literature are 
analysed thoroughly. Moreover, the statistical, threshold, region, and machine learning methods for human skin detection are presented and compared. Furthermore, the specific applications of the reviewed studies are also highlighted. The proposed detection methods in the literature are presented based on their measurement's parameters. It is noted that, each particular application focused on as specific measurement parameter according to the application issue. To the best of our knowledge, a few studies were tried to reduce the detection time. And, the real time human skin detection system is the best target of these studies. There are some researchers calculated real time for skin detection and some papers when they detect skin, they detected face, and hands as well depend on the result time. The remaining researches got result with the minimum is $90 \mathrm{~ms}$ and the maximum is $926.3 \mathrm{~s}$. further investigations is recommended to improve the detection time with maintaining a high accuracy and performance. This review gives an advance view of the human skin detection methods and issues to the researchers.

\section{REFERENCES}

[1] M. R. Mahmoodi and S. M. Sayedi, "A comprehensive survey on human skin detection," International Journal of Image, Graphics \& Signal Processing, vol. 8, no. 5, pp. 1-35, 2016.

[2] S. Naji, H. A. Jalab, and S. A. Kareem, "A survey on skin detection in colored images," Artificial Intelligence Review, vol, 52, no. 2, pp. 1041-1087, 2019.

[3] C. Prema and D. Manimegalai, "Survey on skin tone detection using color spaces," International Journal of Applied Information Systems, vol. 2, no. 2, pp. 18-26, 2012.

[4] B. Martinkauppi, "Face colour under varying illumination: analysis and applications," Dissertation, Department of Electrical and Information Engineering and Infotech Oulu, University of Oulu, 2002.

[5] M. Störring, "Computer vision and human skin colour." Ph.D. Dissertation, Faculty of Engineering and Science, Aalborg University, 2004.

[6] M. Rhanoui, S. Yousfi, M. Mikram, and H. Merizak, "Forecasting financial budget time series: ARIMA random walk vs LSTM neural network," International Journal of Artificial Intelligence, vol. 8, no. 4, pp. 317-327, 2019.

[7] A. Ahmad, M. L. Othman, K. K. B. Zainab, H. Hizam, and N. Azis, "Adaptive ANN based differential protective relay for reliable power transformer protection operation during energisation," International Journal of Artificial Intelligence, vol. 8, no. 4, pp. 307-316, 2019.

[8] W. R. Tan, C. S. Chan, P. Yogarajah, and J. Condell, "A fusion approach for efficient human skin detection,” IEEE Transactions on Industrial Informatics, vol. 8, no. 1, pp. 138-147, Feb. 2012.

[9] S. B. Jadhav, V. R. Udupi, and S. B. Patil, "Convolutional neural networks for leaf image-based plant disease classification," International Journal of Artificial Intelligence, vol. 8, no. 4, pp. 328-341, 2019.

[10] Ming-Hsuan Yang, D. J. Kriegman, and N. Ahuja, "Detecting faces in images: a survey," IEEE Transactions on Pattern Analysis and Machine Intelligence, vol. 24, no. 1, pp. 34-58, Jan. 2002.

[11] P. Kakumanu, S. Makrogiannis, and N. Bourbakis, "A survey of skin-color modeling and detection methods," Pattern Recognition, vol. 40, no. 3, pp. 1106-1122, 2007.

[12] V. Vezhnevets, V. Sazonov, and A. Andreeva, "A survey on pixel-based skin color detection techniques," International Conference Graphicon, vol. 3, pp. 85-92, 2003.

[13] H. K. Al-Mohair, J. Mohamad-Saleh, and S. A. Suandi, "Human skin color detection: A review on neural network perspective," Int. Journal of Innovative Computing, Information and Control, vol. 8, no. 12, pp. 8115-8131, 2012.

[14] A. Martínez and R. Benavente, "The AR face database, 1998," Comp. Vision Center, Tech. Report, vol. 3, no. 5, 2007.

[15] P. Dollar, C. Wojek, B. Schiele, and P. Perona, "Pedestrian detection: A benchmark," 2009 IEEE Conference on Computer Vision and Pattern Recognition, pp. 304-311, 2009.

[16] R. Gross, I. Matthews, J. Cohn, T. Kanade, and S. Baker, "Multi-pie," Image and Vision Computing, vol. 28, no. 5, pp. 807-813, 2010.

[17] M. J. Jones and J. M. Rehg, "Statistical color models with application to skin detection," International Journal of Computer Vision, vol. 46, no. 1, pp. 81-96, 2002.

[18] S. L. Phung, A. Bouzerdoum, and D. Chai, "Skin segmentation using color pixel classification: analysis and comparison," IEEE Transactions on Pattern Analysis and Machine Intelligence, vol. 27, no. 1, pp. 148-154, 2005.

[19] M. Everingham, L. Van Gool, C. K. I. Williams, J. Winn, and A. Zisserman, "The pascal visual object classes (VOC) challenge,” International Journal of Computer Vision, vol. 88, no. 2, pp. 303-338, 2010.

[20] P. J. Phillips, Hyeonjoon Moon, P. Rauss, and S. A. Rizvi, "The FERET evaluation methodology for facerecognition algorithms," Proc. of IEEE Comp. Society Conf. on Comp. Vision and Patt. Recog., pp. 137-143. 1997.

[21] M. Kawulok, J. Kawulok, and J. Nalepa, "Spatial-based skin detection using discriminative skin-presence features," Pattern Recognition Letters, vol. 41, pp. 3-13, 2014.

[22] Q. Zhu, C-T. Wu, K-T. Cheng, and Y.-L. Wu, "An adaptive skin model and its application to objectionable image filtering," in Proceedings of the 12th annual ACM international conference on Multimedia, pp. 56-63, 2004.

[23] G. B. Huang, M. Mattar, T. Berg, and E. Learned-Miller, "Labeled faces in the wild: A database for studying face recognition in unconstrained environments," Works. on Faces in 'Real-Life' Imag.: Detec., Align., and Recog., 2008.

[24] J. P. B. Casati, D. R. Moraes, and E. L. L. Rodrigues, "SFA: A human skin image database based on FERET and AR facial images," IX workshop de Visao Computational, Rio de Janeiro, 2013.

[25] P. Sharma and R. B. Reilly, "A colour face image database for benchmarking of automatic face detection algorithms," Proceedings EC-VIP-MC 2003. 4th EURASIP Conference focused on Video/Image Processing and Multimedia Communications (IEEE Cat. No.03EX667), vol. 1, pp. 423-428, 2003. 
[26] S. L. Phung, A. Bouzerdoum, and D. Chai, "Skin segmentation using color and edge information," Proc. Seventh International Symposium on Signal Processing and Its Applications, vol. 1, pp. 525-528, 2003.

[27] A. K. Jain, "Fundamentals of digital image processing," Prentice-Hall, Inc., 1989.

[28] J. C. Russ, "Introduction to image processing and analysis," CRC press, 2007.

[29] S. Roheda, "A multi-scale approach to skin pixel detection," Electronic Imaging, vol. 2017, no. 4, pp. 18-23, 2017.

[30] Rein-Lien Hsu, M. Abdel-Mottaleb, and A. K. Jain, "Face detection in color images," IEEE Transactions on Pattern Analysis and Machine Intelligence, vol. 24, no. 5, pp. 696-706, May 2002.

[31] K-M. Cho, J-H. Jang, and K-S. Hong, "Adaptive skin-color filter," Patt. Recog., vol. 34, no. 5, pp. 1067-1073, 2001.

[32] S. Bilal, R. Akmeliawati, M. J. E. Salami, and A. A. Shafie, "Dynamic approach for real-time skin detection," Journal of Real-Time Image Processing, vol. 10, no. 2, pp. 371-385, 2015.

[33] M. R. Mahmoodi, "Fast and efficient skin detection for facial detection," arXiv Prepr. arXiv1701.05595, 2017.

[34] M. Sharif, S. Mohsin, and M. Y. Javed, "Real time face detection using skin detection (Block Approach)," Journal of Applied Computer Science \& Mathematics, vol. 10, no. 5, pp. 75-81, 2011.

[35] Saranya S. and Keerthikumar D. N., "Real-time skin detection and tracking based on FPGA," International Journal of Engineering Technology, Management and Applied Sciences, vol. 4, no. 12, pp. 90-97, 2016.

[36] K. A. Kaabneh, "Reliable skin detection using hybrid neural network model," International Journal of Advanced Research in Computer and Communication Engineering, vol. 3, no. 3, pp. 5802-5805, 2014.

[37] Y-H. Chen, K-T. Hu, and S-J. Ruan, "Statistical skin color detection method without color transformation for realtime surveillance systems," Engineering Applications of Artificial Intelligence, vol. 25, no. 7, pp. 1331-1337, 2012.

\section{BIOGRAPHIES OF AUTHORS}

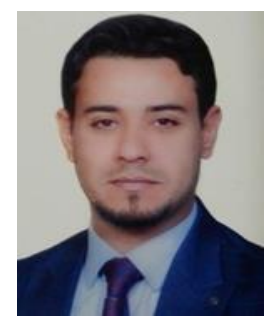

Hussein Ali Hussein Al Naffakh received the B.Sc. in computer science from University of Kufa/ Faculty of Education in (2014)- Iraq; He is doing M.Sc. (Computer Science) in Software Division from University of Babylon/ College of Information Technology in 2017. Ph.D. candidate in Information Technology at Universiti Tun Hussein Onn Malaysia (UTHM). He is interested in the following field (Steganography, Cryptology, Information security and Information system, image processing, artificial intelligence).

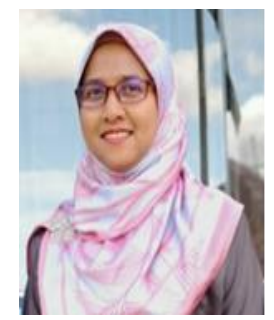

Rozaida Ghazali is currently a Professor at the Faculty of Computer Science and Information Technology, Universiti Tun Hussein Onn Malaysia (UTHM). She graduated with a Ph.D. degree in Higher Order Neural Networks from the School of Computing and Mathematical Sciences at Liverpool John Moores University, United Kingdom in 2007. Earlier, in 2003 she completed her M.Sc. degree in Computer Science from Universiti Teknologi Malaysia (UTM). She received her B.Sc. (Hons) degree in Computer Science from Universiti Sains Malaysia (USM) in 1997. In 2001, Rozaida joined the academic staff in UTHM. Her research area includes neural networks, swarm intelligence, optimization, data mining, and time series prediction. She has successfully supervised a number of $\mathrm{PhD}$ and master students and published more than 150 articles in various international journals and conference proceedings. She acts as a reviewer for various journals and conferences. She has also served as a conference chair, and as a technical committee for numerous international conferences.

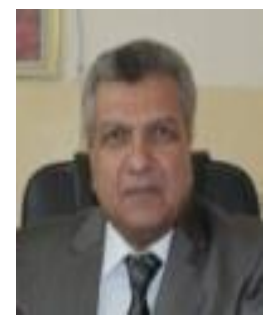

Nidhal Khdhair El Abbadi received B.Sc. in Chemical Engineering, B.Sc. in computer science, M.Sc. and Ph.D. in computer science, worked in industry and many universities, reviewer for a number of international journals, has many published papers and six published books (Programming with Pascal, C++ from beginning to OOP, Data structures in C++, Principles of Computer Graphics, effect of modern technology on talent, and chapter in Handbook of Research on Threat Detection and Countermeasures in Network Security book), his research interests are in image processing, security, and steganography, He's Professor in Computer Science in the University of Kufa - Najaf, IRAQ. 